\title{
On the smallest singular value in the class of unit lower triangular matrices with entries in $[-a, a]$
}

https://doi.org/10.1515/spma-2020-0139

Received October 31, 2020; accepted May 24, 2021

Abstract: Given a real number $a \geq 1$, let $K_{n}(a)$ be the set of all $n \times n$ unit lower triangular matrices with each element in the interval $[-a, a]$. Denoting by $\lambda_{n}(\cdot)$ the smallest eigenvalue of a given matrix, let $c_{n}(a)=$ $\min \left\{\lambda_{n}\left(Y Y^{T}\right): Y \in K_{n}(a)\right\}$. Then $\sqrt{c_{n}(a)}$ is the smallest singular value in $K_{n}(a)$. We find all minimizing matrices. Moreover, we study the asymptotic behavior of $c_{n}(a)$ as $n \rightarrow \infty$. Finally, replacing $[-a, a]$ with $[a, b], a \leq 0<b$, we present an open question: Can our results be generalized in this extension?

Keywords: real symmetric matrix, unit lower triangular matrix, GCD and LCM matrix, smallest eigenvalue, smallest singular value

MSC: 11C20, 15A18, 15A42, 15B36

\section{Introduction}

Let $S=\left\{x_{1}, x_{2}, \ldots, x_{n}\right\}$ be a set of distinct positive integers and let $\left(x_{i}, x_{j}\right)$ and $\left[x_{i}, x_{j}\right]$ denote the greatest common divisor and the least common multiply of $x_{i}$ and $x_{j}$, respectively. The $n \times n$ matrices $(S)=\left(\left(x_{i}, x_{j}\right)\right)$ and $[S]=\left(\left[x_{i}, x_{j}\right]\right)$ are called the GCD matrix and the LCM matrix on $S$, respectively. Many results on these matrices, their various generalizations and relatives have been published in the literature. For general accounts, see $[2-4,6,8,12]$ and the references therein.

One of the richest topics in the study of GCD and LCM matrices is their spectral properties. In this frame, Hong and Loewy [6] studied the asymptotic behavior of the eigenvalues of power GCD matrices and introduced a new parameter to present a lower bound for the smallest eigenvalue of the power GCD matrix. They defined the numbers $c_{n}$ depending only on $n$ as follows:

$$
c_{n}=\min \left\{\lambda_{n}\left(Y Y^{T}\right): Y \in K_{n}\right\},
$$

where $\lambda_{n}(\cdot)$ is the smallest eigenvalue and $K_{n}$ is the set of all $n \times n$ nonsingular lower triangular $(0,1)$-matrices. Then, in the light of their MATLAB calculations for $n=2,3, \ldots, 7$, Ilmonen, Haukkanen and Merikoski [8] conjectured that $c_{n}(a)=\lambda_{n}\left(Y_{0} Y_{0}^{T}\right)$, where $Y_{0}=\left(y_{i j}^{0}\right) \in K_{n}$ is defined by $y_{i j}^{0}=\frac{1-(-1)^{i+j}}{2}$ if $i>j$. Verifying the truth of the conjecture for $n=8$ and 9 in [1], the author of the present paper, Keskin, Yıldız and Demirbüken [4] proved the conjecture and realized that there is only one matrix $Y \in K_{n}$ for which $c_{n}$ is attained. Therefore, it was conjectured that if $c_{n}=\lambda_{n}\left(Y Y^{T}\right)$ for $Y \in K_{n}$, then $Y=Y_{0}$ [4, Conjecture 3.1]. Recently, Loewy [11] has proved the conjecture.

Due to the importance of the study of spectral properties of GCD and related matrices, some authors tried to find bounds for $c_{n}$. In this frame, assuming the truth of the conjecture, Mattila [12] obtained the following

^Corresponding Author: Ercan Altınışık: Department of Mathematics, Faculty of Sciences, Gazi University 06500 Teknikokullar - Ankara, Turkey, E-mail: ealtinisik@gazi.edu.tr 
lower bound

$$
\begin{gathered}
c_{n} \geq\left(\frac{48}{n^{4}+56 n^{2}+48 n}\right)^{\frac{n-1}{2}} \text { for even } n, \\
c_{n} \geq\left(\frac{48}{n^{4}+50 n^{2}+48 n-51}\right)^{\frac{n-1}{2}} \text { for odd } n .
\end{gathered}
$$

Beside this, Merikoski, who contributed to the paper [4], improved Mattila's lower bound as follows:

$$
c_{n} \geq \frac{2}{2 F_{n} F_{n+1}+1+(-1)^{n}},
$$

where $F_{n}$ is the $n$th Fibonacci number. In addition to these bounds, in [2] the inequality

$$
c_{n} \geq\left(n \sum_{k=1}^{n} \mu^{2}(k)\right)^{-1}
$$

where $\mu$ is the Möbius function, was proposed for $c_{n}$ by using a different method. Kaarnioja [9] improved the lower bound further and showed that

$c_{n} \geq\left(\frac{1}{25} \varphi^{-4 n}+\frac{3+(-1)^{n}}{25} \varphi^{-2 n}-\frac{2}{5 \sqrt{5}} n \varphi^{-2 n}+\frac{13(-1)^{n}-33}{50}+n+\frac{3+(-1)^{n}}{25} \varphi^{2 n}+\frac{2}{5 \sqrt{5}} n \varphi^{2 n}+\frac{1}{25} \varphi^{4 n}\right)^{-\frac{1}{2}}$,

where $\varphi=\frac{1+\sqrt{5}}{2}$ is the golden ratio. He also conjectured asymptotically that

$$
c_{n} \sim \frac{5}{\varphi^{2 n}}
$$

which was proved by Loewy [11].

This paper has two main goals. The first is to expand the existing results on the process of finding all matrices for which $\sqrt{c_{n}}$ is attained. The second is to determine the asymptotic behaviour of $c_{n}$ in a larger class of matrices. Given a real number $a \geq 1$, let $K_{n}(a)$ denote the set of all $n \times n$ unit lower triangular matrices whose each element under diagonal is in the interval $[-a, a]$. We note that all diagonal entries of a unit lower triangular matrix are 1 . Let

$$
c_{n}(a)=\min \left\{\lambda_{n}\left(Y Y^{T}\right): Y \in K_{n}(a)\right\},
$$

where $\lambda_{n}(\cdot)$ is the smallest eigenvalue. It is clear that $\sqrt{c_{n}(a)}$ is the smallest singular value in $K_{n}(a)$. Let $Y_{0}=\left(y_{i j}^{0}\right) \in K_{n}(a)$ be defined by

$$
y_{i j}^{0}=(-1)^{i+j+1} a
$$

for $i>j$. In Section 2, we present a sharp upper bound for the absolute values of entries of $Y^{-1}$ and $\left(Y Y^{T}\right)^{-1}$, where $Y \in K_{n}(a)$, and obtain all the entries of $Y_{0}^{-1}$ and $\left(Y_{0} Y_{0}^{T}\right)^{-1}$ in terms of $a$. In Section 3, we prove that $c_{n}(a)=\lambda_{n}\left(Y_{0} Y_{0}^{T}\right)$. We also show that if $c_{n}(a)=\lambda_{n}\left(Y Y^{T}\right)$ for $Y \in K_{n}(a)$ then $Y=E Y_{0} E$, where $E=\operatorname{diag}\left(\varepsilon_{1}, \varepsilon_{2}, \ldots, \varepsilon_{n}\right)$ with $\varepsilon_{i}= \pm 1$ for $i=1,2, \ldots, n$. Moreover, we determine the asymptotic behavior of $c_{n}(a)$. In other words, we show that $c_{n}(a) \sim \frac{(a+2)^{2}}{(a+1)^{2 n}-1}$ as $n \rightarrow \infty$. Finally, we present concluding remarks in Section 4, including some open problems related to a certain generalization of the results on $c_{n}$ and $c_{n}(a)$.

\section{Preliminaries}

In order to find the bound mentioned in the introduction, we present five lemmas here. In the first lemma, we obtain a recurrence relation for the entries of inverses of matrices in $K_{n}(a)$.

Lemma 1. Let $Y \in K_{n}(a), W=\left(w_{i j}\right)=Y-I$ and $Y^{-1}=\left(b_{i j}\right)$. Then,

$$
b_{i j}=\left\{\begin{array}{cl}
0 & \text { if } i<j, \\
1 & \text { if } i=j, \\
-\sum_{k=j}^{i-1} w_{i k} b_{k j} & \text { if } i>j .
\end{array}\right.
$$


Proof. See [4, Lemma 2.2]. The set of matrices is different, but the proof works also here.

Lemma 2. [5] Let $Y$ and $Y^{-1}$ be as in Lemma 1. Then,

$$
\left|b_{i j}\right| \leq a(a+1)^{i-j-1}
$$

for $1 \leq j<i \leq n$.

Proof. Let $W=\left(w_{i j}\right)$ be as in Lemma 1. Let $j=1,2, \ldots, n-1$. We prove by induction on $t=1,2, \ldots, n-j$ that $\left|b_{j+t, j}\right| \leq a(a+1)^{t-1}$. By Lemma 1, we have $\left|b_{j+1, j}\right|=\left|w_{j+1, j}\right|$, where $w_{j+1, j} \in[-a, a]$. Thus, $\left|b_{j+1, j}\right| \leq a$. Now assume that the inequality $\left|b_{j+t, j}\right| \leq a(a+1)^{t-1}$ holds for each $t=1,2, \ldots, k-1$. We show that $\left|b_{j+k, j}\right| \leq$ $a(a+1)^{k-1}$. By Lemma 1 and the induction hypothesis, we have

$$
\begin{aligned}
\left|b_{j+k, j}\right| & =\left|\sum_{i=j}^{j+k-1} w_{j+k, i} b_{i j}\right| \\
& \leq \sum_{i=j}^{j+k-1}\left|w_{j+k, i}\right|\left|b_{i j}\right| \\
& \leq a \sum_{i=j}^{j+k-1}\left|b_{i j}\right| \\
& \leq a\left(1+\sum_{i=j+1}^{j+k-1} a(a+1)^{i-j-1}\right) \\
& =a(a+1)^{k-1} .
\end{aligned}
$$

The induction principle completes the proof.

Lemma 3. [5] Let $Y_{0}$ be as in (2) and $Y_{0}^{-1}=\left(c_{i j}\right)$. Then

$$
c_{i j}=\left\{\begin{array}{cl}
0 & \text { if } i<j, \\
1 & \text { if } i=j, \\
(-1)^{i-j} a(a+1)^{i-j-1} & \text { if } i>j .
\end{array}\right.
$$

Proof. Let $W_{0}=\left(w_{i j}^{0}\right)=Y_{0}-I$. Then

$$
w_{i j}^{0}=\left\{\begin{array}{cl}
0 & \text { if } i \leq j \\
(-1)^{i+j+1} a & \text { otherwise }
\end{array}\right.
$$

Since $Y_{0} \in K_{n}(a)$, we have by Lemma 1

$$
c_{i j}=-\sum_{k=j}^{i-1} w_{i k}^{0} c_{k j}
$$

for $i>j$.

Next, we show by induction on $t=i-j$ that

$$
c_{i j}=(-1)^{i-j} a(a+1)^{i-j-1}, \quad i>j .
$$

If $t=1$, then

$$
c_{j+1, j}=-w_{j+1, j}^{0}=-a .
$$

Now assume that (3) holds for $t=1,2, \ldots, k-1$. Then

$$
c_{j+k, j}=-\sum_{s=j}^{j+k-1} w_{j+k, s}^{0} c_{s j}=(-1)^{k} a+\sum_{s=j+1}^{j+k-1}(-1)^{j+k+s} a(-1)^{s-j} a(a+1)^{s-j-1}=(-1)^{k} a(a+1)^{k-1} .
$$

The induction principle completes the proof. 
In the following, we denote by $(\cdot)_{i j}$ the $i j$-th entry of a given matrix.

Lemma 4. [5] Let $Y_{0}$ be as in (2) and $Z_{0}=Y_{0} Y_{0}^{T}$. Then,

$$
\left(Z_{0}^{-1}\right)_{i j}=\left\{\begin{array}{cl}
1+\frac{a^{2}}{(a+1)^{2}-1}\left((a+1)^{2(n-i)}-1\right) & \text { if } i=j, \\
(-1)^{|i-j|}\left(a(a+1)^{|i-j|-1}+\frac{a^{2}}{(a+1)^{2}-1}(a+1)^{|i-j|}\left((a+1)^{2(n-\max (i, j))}-1\right)\right) & \text { if } i \neq j .
\end{array}\right.
$$

Proof. By Lemma 3, we have

$$
\left(Z_{0}^{-1}\right)_{i i}=1+\sum_{k=i+1}^{n} a^{2}(a+1)^{2(k-i-1)}=1+\frac{a^{2}}{(a+1)^{2}-1}\left((a+1)^{2(n-i)}-1\right)
$$

for all $i=1,2, \ldots, n$ and

$$
\begin{aligned}
\left(Z_{0}^{-1}\right)_{i j} & =c_{j i}+\sum_{t=j+1}^{n} c_{t i} c_{t j} \\
& =(-1)^{j-i}\left(a(a+1)^{j-i-1}+\sum_{t=j+1}^{n} a^{2}(a+1)^{2 t-i-j-2}\right) \\
& =(-1)^{j-i}\left(a(a+1)^{j-i-1}+a^{2}(a+1)^{j-i} \frac{(a+1)^{2(n-j)}-1}{(a+1)^{2}-1}\right)
\end{aligned}
$$

for $1 \leq i<j \leq n$. Since $Z_{0}$ is symmetric, the claim follows.

Lemma 5. [5] Let $Z=Y Y^{T}, Y \in K_{n}(a)$, and let $Z_{0}$ be as in Lemma 4. Then $\left|Z^{-1}\right| \leq\left|Z_{0}^{-1}\right|$, that is $\left|\left(Z^{-1}\right)_{i j}\right| \leq\left|\left(Z_{0}^{-1}\right)_{i j}\right|$ for all $1 \leq i, j \leq n$.

Proof. Let $Y^{-1}$ be as in Lemma 1. Then, by Lemmas 2 and 4,

$$
\begin{aligned}
\left|\left(Z^{-1}\right)_{i i}\right| & =\left|\sum_{k=1}^{n} b_{k i}^{2}\right| \\
& =\sum_{k=1}^{n}\left|b_{k i}\right|^{2} \\
& =\sum_{k=i}^{n}\left|b_{k i}\right|^{2} \\
& \leq 1+\sum_{k=i+1}^{n} a^{2}(a+1)^{2(k-i-1)} \\
& =\left|\left(Z_{0}^{-1}\right)_{i i}\right|
\end{aligned}
$$

for all $i=1,2, \ldots, n$. Let $i<j$. Again, by Lemmas 2 and 4 ,

$$
\begin{aligned}
\left|\left(Z^{-1}\right)_{i j}\right| & =\left|\sum_{t=1}^{n} b_{t i} b_{t j}\right| \\
& \leq \sum_{t=1}^{n}\left|b_{t i}\right|\left|b_{t j}\right| \\
& =\left|b_{j i}\right|+\sum_{t=j+1}^{n}\left|b_{t i}\right|\left|b_{t j}\right| \\
& \leq a(a+1)^{j-i-1}+\sum_{t=j+1}^{n} a^{2}(a+1)^{2 t-i-j-2}
\end{aligned}
$$




$$
=\left|\left(Z_{0}^{-1}\right)_{i j}\right| \cdot
$$

Since $Z^{-1}$ and $Z_{0}^{-1}$ are symmetric, we obtain $\left|Z^{-1}\right| \leq\left|Z_{0}^{-1}\right|$.

\section{Main Results}

Theorem 1. Let $Y_{0}$ be as in (2). Then $c_{n}(a)=\lambda_{n}\left(Y_{0} Y_{0}^{T}\right)$. Conversely, if $Y \in K_{n}(a)$ and $\lambda_{n}\left(Y Y^{T}\right)=c_{n}(a)$, then $Y=E Y_{0} E$, where $E=\operatorname{diag}\left(\varepsilon_{1}, \varepsilon_{2}, \ldots, \varepsilon_{n}\right)$ with $\varepsilon_{i}= \pm 1$ for $i=1,2, \ldots, n$.

Proof. For the first claim, we proceed as in the proof [4] of the Ilmonen-Haukkanen-Merikoski conjecture. Let $Z_{0}=Y_{0} Y_{0}^{T}$. Then, by (4) and (5), $\operatorname{tr} Z_{0}^{-k}=\operatorname{tr}\left|Z_{0}^{-1}\right|^{k}$ for all positive integers $k$. By Newton's identities [10], one can easily see that $Z_{0}^{-1}$ and $\left|Z_{0}^{-1}\right|$ have the same characteristic polynomial. Since they are real symmetric matrices, their spectral radii are equal, that is, $\rho\left(\left|Z_{0}^{-1}\right|\right)=\rho\left(Z_{0}^{-1}\right)$. Let $Y \in K_{n}(a)$ and $Z=Y Y^{T}$. By Lemma 5 and [7, Theorem 8.1.18], we obtain the following inequalities

$$
\rho\left(Z^{-1}\right) \leq \rho\left(\left|Z^{-1}\right|\right) \leq \rho\left(\left|Z_{0}^{-1}\right|\right)=\rho\left(Z_{0}^{-1}\right) .
$$

Since $Z^{-1}$ and $Z_{0}^{-1}$ are positive definite, we have

$$
\rho\left(Z^{-1}\right)=\lambda_{1}\left(Z^{-1}\right) \quad \text { and } \quad \rho\left(Z_{0}^{-1}\right)=\lambda_{1}\left(Z_{0}^{-1}\right)
$$

where $\lambda_{1}(\cdot)$ is the largest eigenvalue. Hence, by (8),

$$
\lambda_{n}(Z)=\frac{1}{\lambda_{1}\left(Z^{-1}\right)} \geq \frac{1}{\lambda_{1}\left(Z_{0}^{-1}\right)}=\lambda_{n}\left(Z_{0}\right)
$$

and the claim follows.

To show that the second claim, let $Y \in K_{n}(a)$ satisfy $c_{n}(a)=\lambda_{n}(Z), Z=Y Y^{T}$. Then $\lambda_{n}(Z)=\lambda_{n}\left(Z_{0}\right)$ and further $\rho\left(Z^{-1}\right)=\rho\left(Z_{0}^{-1}\right)$, which implies by (8) that $\rho\left(\left|Z^{-1}\right|\right)=\rho\left(\left|Z_{0}^{-1}\right|\right)$. Now, by the same reasoning as in the proof of [11, Theorem 3.1], we have

$$
\left|Z^{-1}\right|=\left|Z_{0}^{-1}\right| \text {. }
$$

Let $Y^{-1}=\left(b_{i j}\right)$ and $Y_{0}^{-1}=\left(c_{i j}\right)$. Since $Y^{-1}$ is a lower triangular matrix, inequalities (6) and (7), and equality (9) together imply that

$$
\left|\sum_{k=j}^{n} b_{k i} b_{k j}\right|=\sum_{k=j}^{n}\left|b_{k i}\right|\left|b_{k j}\right|
$$

for $1 \leq i<j \leq n$. Here, all the summands are nonzero. Note that all the summands on the left-hand side of the last equality have the same argument. Furthermore, we have by Lemmas 2 and 3 that

$$
b_{i j}=\epsilon_{i, j} c_{i j} \text {, }
$$

where $\epsilon_{i, j}= \pm 1$ and $\epsilon_{i, i}=1$ for all $1 \leq i, j \leq n$. Then,

$$
\begin{aligned}
\left|\sum_{k=j}^{n} \epsilon_{k, i} \epsilon_{k, j} c_{k i} c_{k j}\right|=\sum_{k=j}^{n}\left|\epsilon_{k, i} c_{k i}\right|\left|\epsilon_{k, j} c_{k j}\right| & \Longleftrightarrow\left|\sum_{k=j}^{n} \epsilon_{k, i} \epsilon_{k, j} c_{k i} c_{k j}\right|=\sum_{k=j}^{n}\left|c_{k i}\right|\left|c_{k j}\right| \\
& \Longleftrightarrow \epsilon_{j, i}=\epsilon_{j+1, i} \epsilon_{j+1, j}=\epsilon_{j+2, i} \epsilon_{j+2, j}=\cdots=\epsilon_{n, i} \epsilon_{n, j}
\end{aligned}
$$

for $1 \leq i<j \leq n$. This means that every $\epsilon_{j, i}$ depends only $\epsilon_{n, i}$ and $\epsilon_{n, j}$. Therefore, setting $\varepsilon_{i}=\epsilon_{n, i}$ for all $i=1,2, \ldots, n$, for the sake of brevity, we have

$$
b_{i j}=\varepsilon_{i} \varepsilon_{j} c_{i j}
$$


or equivalently

$$
Y^{-1}=E Y_{0}^{-1} E^{-1},
$$

where $E=\operatorname{diag}\left(\varepsilon_{1}, \varepsilon_{2}, \ldots, \varepsilon_{n}\right)$. It is clear that $E=E_{1} E_{2} \ldots E_{n}$, where each $E_{i}(i=1,2, \ldots, n)$ is an elementary matrix obtained from the $n \times n$ identity matrix by multiplying its $i$ th row by $\varepsilon_{i}$. So, $E=E^{-1}$. and therefore $Y=E Y_{0} E$. This concludes the proof.

Theorem 2. We have

$$
\frac{1}{n-\frac{a n}{a+2}+\frac{(a+1)^{2 n}-1}{(a+2)^{2}}} \leq c_{n}(a) \leq \frac{1}{1-\frac{a n}{a+2}+\frac{(a+1)^{2 n}-1}{(a+2)^{2}}}
$$

and consequently

$$
\lim _{n \rightarrow \infty} \frac{c_{n}(a)}{\frac{(a+2)^{2}}{(a+1)^{2 n}-1}}=1
$$

Proof. To prove the theorem, we use a similar but not the same method to those of Theorem 2.1 in [11].

First, let $Z_{0}$ be as in Lemma 4 and $H_{n}=I-Z_{0}^{-1}$. Now, add $a+1$ multiple of the second column to the first column, and then add $a+1$ multiple of the second row to the first row. Let $M_{n}=\left(m_{i j}\right)$ be the matrix obtained by these two elementary operations from $H_{n}$. It is clear that $\operatorname{det}\left(H_{n}\right)=\operatorname{det}\left(M_{n}\right), m_{11}=a(a+2), m_{12}=m_{21}=a$ and $m_{1 j}=m_{j 1}=0$ for $j=3,4, \ldots, n$. By expanding $\operatorname{det}\left(M_{n}\right)$, one can easily obtain the following recurrence relation for $n \geq 3$,

$$
\operatorname{det}\left(H_{n}\right)=a(a+2) \operatorname{det}\left(H_{n-1}\right)-a^{2} \operatorname{det}\left(H_{n-2}\right)
$$

with the initial conditions $\operatorname{det}\left(H_{1}\right)=0$ and $\operatorname{det}\left(H_{2}\right)=-a^{2}$.

Second, we show by induction on $n$ that $\operatorname{det}\left(H_{n+1}\right)<\operatorname{det}\left(H_{n}\right)$ for $n>1$. It is clear that $\operatorname{det}\left(H_{2}\right)<\operatorname{det}\left(H_{1}\right)=$ $-a^{2}$. Suppose $\operatorname{det}\left(H_{n}\right)<\operatorname{det}\left(H_{n-1}\right)$. Then,

$$
\begin{aligned}
\operatorname{det}\left(H_{n+1}\right) & =a(a+2) \operatorname{det}\left(H_{n}\right)-a^{2} \operatorname{det}\left(H_{n-1}\right) \\
& <a(a+2) \operatorname{det}\left(H_{n}\right)-a^{2} \operatorname{det}\left(H_{n}\right) \\
& =2 a \operatorname{det}\left(H_{n}\right) \\
& <\operatorname{det}\left(H_{n}\right)
\end{aligned}
$$

by the induction hypothesis and the assumption $a \geq 1$. Thus, we have $\operatorname{det}\left(H_{n+1}\right)<\operatorname{det}\left(H_{n}\right)<0$ for all $n>1$.

Next, we show that every eigenvalue of $Z_{0}^{-1}$, except its spectral radius $\rho\left(Z_{0}^{-1}\right)$, lies in the interval $[0,1]$. Let $\lambda_{1}^{(n)} \geq \lambda_{2}^{(n)} \geq \cdots \geq \lambda_{n}^{(n)}$ be the eigenvalues of the $n \times n$ matrix $Z_{0}^{-1}$. It is clear that $\lambda_{n}^{(n)}>0$ as $Z_{0}^{-1}$ is positive definite. We show again by induction on $n$ that $\lambda_{1}^{(n)}>1$ and $\lambda_{2}^{(n)}<1$. For $n=2$,

$$
\lambda_{1}^{(n)}=\frac{a^{2}+2+\sqrt{\left(a^{2}+2\right)^{2}-4}}{2}>1 \text { and } \lambda_{2}^{(n)}=\frac{a^{2}+2-\sqrt{\left(a^{2}+2\right)^{2}-4}}{2}<1 .
$$

Assume $\lambda_{1}^{(n-1)}>1$ and $\lambda_{2}^{(n-1)}<1$. Then it follows from the Cauchy interlacing inequalities, see [7], that $\lambda_{3}^{(n)} \leq$ $\lambda_{2}^{(n-1)} \leq 1$. Note that $\operatorname{det}\left(I-Z_{0}^{-1}\right)=\operatorname{det}\left(H_{n}\right)<0$ from paragraph above. Moreover, the characteristic polynomial of $Z_{0}^{-1}$, evaluated at $x=1$ is negative. So, we obtain $\lambda_{2}^{(n)}<1$. Thus, it follows that

$$
\rho\left(Z_{0}^{-1}\right) \leq \operatorname{tr}\left(Z_{0}^{-1}\right) \leq \rho\left(Z_{0}^{-1}\right)+n-1 .
$$

In addition to this, by (4), one can easily calculate the trace of $Z_{0}^{-1}$ as

$$
\begin{aligned}
\operatorname{tr}\left(Z_{0}^{-1}\right) & =\sum_{i=1}^{n}\left(1+\frac{a^{2}}{(a+1)^{2}-1}\left((a+1)^{2(n-i)}-1\right)\right) \\
& =n-\frac{a n}{a+2}+\frac{(a+1)^{2 n}-1}{(a+2)^{2}} .
\end{aligned}
$$

So, it is obvious that $\operatorname{tr}\left(Z_{0}^{-1}\right)-n+1>0$. The first result given in (10) follows from the inequalities in (12) and the fact $c_{n}(a)=\rho\left(Z_{0}^{-1}\right)^{-1}$. For the second result, we restate the inequalities in (10) as

$$
\frac{1}{2(a+2) \frac{n}{(a+1)^{2 n}-1}+1} \leq \frac{c_{n}(a)}{\frac{(a+2)^{2}}{(a+1)^{2 n}-1}} \leq \frac{1}{\frac{a+2}{a} \cdot \frac{-a^{2} n+(a+1)^{2}-1}{(a+1)^{2 n}-1}+1} .
$$


Thus, the claim in (11) follows.

\section{Concluding Remarks}

In this paper, introducing a new constant $c_{n}(a)$, we have expanded the results on the finding all minimizing matrices of the constant $c_{n}$ and its asymptotic behaviour to a larger class of matrices. We do not reckon that our constant $c_{n}(a)$ could be used in eigenvalue estimation of GCD and related matrices as $c_{n}$ was used in the literature, see $[3,6,8,9,11,12]$. However, it seems possible that the techniques of this paper could be applied to a larger class of matrices than those considered in this present paper.

Throughout the paper, we assumed that $a \geq 1$. Almost all of our results are valid for $a>0$. However, in this case, it seems not easy to prove that $\operatorname{det}\left(H_{n}\right)<0$ for $n>1$, and the lower and upper bounds for $c_{n}(a)$ given in (10) for small values of $n$ do not provide good estimates. For example, when we compute the three terms in the inequalities in (10) for $a=0.1$ and $n=5$, we find the numbers

$$
0.195186787128691,0.657178123193002,0.890236025695808,
$$

respectively. Also

$$
\frac{(a+2)^{2}}{(a+1)^{2 n}-1} \approx 2.76707191431876 .
$$

On the other hand, when $a \geq 1$, we have better lower and upper bounds for $c_{n}(a)$ even for small $a$ and $n$. Indeed, computing the three terms in (10) for $a=3$ and $n=5$, we find the numbers

$$
0.0000238407438312075,0.00002384095983146484,0.0000238430175723040 \text {, }
$$

respectively. Moreover,

$$
\frac{(a+2)^{2}}{(a+1)^{2 n}-1} \approx 0.0000238418806475455 \text {. }
$$

In addition to this, we believe that the results concerning $c_{n}(a)$ could be generalized to the interval $[a, b]$, where $a \leq 0$ and $b>0$. Let $K_{n}[a, b]$ denote the set of all $n \times n$ unit lower triangular matrices with each element in the interval $[a, b]$. Let $c_{n}(a, b)=\min \left\{\lambda_{n}\left(Y Y^{T}\right): Y \in K_{n}[a, b]\right\}$, i.e., $\sqrt{c_{n}(a, b)}$ is the smallest singular value in $K_{n}[a, b]$. We end the paper by raising the following open problems: Can one determine such a matrix $Y$ in $K_{n}[a, b]$ ? Is it possible to determine the asymptotic behavior of $c_{n}(a, b)$ ?

Acknowledgement: The author would like to thank the anonymous referees for their valuable remarks and corrections which helped to improve the manuscript.

Data Availability Statement: Data sharing is not applicable to this article as no datasets were generated or analysed during the current study.

\section{References}

[1] E. Altınışık, On a conjecture on the smallest eigenvalues of some special positive definite matrices, 3rd International Conference on Applied Mathematics \& Approximation Theory - AMAT 2015, 28-31 May 2015, Ankara, Turkey.

[2] E. Altınışık and Ş. Büyükköse, A proof of a conjecture on monotonic behavior of the smallest and the largest eigenvalues of a number theoretic matrix, Linear Algebra Appl. 471, 141-149 (2015).

[3] E. Altınışık and Ş. Büyükköse, On bounds for the smallest and the largest eigenvalues of GCD and LCM matrices, Math. Inequal. Appl. 19, 117-125 (2016).

[4] E. Altınışık, A. Keskin, M. Yıldız and M. Demirbüken, On a conjecture of Ilmonen, Haukkanen and Merikoski concerning the smallest eigenvalues of certain GCD related matrices, Linear Algebra Appl. 493, 1-13 (2016).

[5] E. Altınışık, A. Keskin and M. Yıldız, A note on the smallest eigenvalues of a certain class of matrices, unpublished preprint, 5 pages (2016). 
[6] S. Hong and R. Loewy, Asymptotic behavior of eigenvalues of greatest common divisor matrices, Glasg. Math. J. 46, 551-569 (2004).

[7] R. Horn and C. R. Johnson, Matrix Analysis, second ed. (Cambridge University Press, Cambridge, London, 2013).

[8] P. Ilmonen, P. Haukkanen and J. K. Merikoski, On eigenvalues of meet and join matrices associated with incidence functions, Linear Algebra Appl. 429, 859-874 (2008).

[9] V. Kaarnioja, Bounds on the spectrum of nonsingular triangular (0, 1)-matrices, J. Combin. Theory, Ser. A 178, 105353,14 pages (2021).

[10] D. Kalman, A matrix proof of Newton's identities, Math. Mag. 73, 313-315 (2000).

[11] R. Loewy, On the smallest singular value in the class of invertible lower triangular $(0,1)$ matrices, Linear Algebra Appl. 608, 203-213 (2021).

[12] M. Mattila, On the eigenvalues of combined meet and join matrices, Linear Algebra Appl. 466, 1-20 (2015). 